Europhys. Lett., 49 (6), pp. 814-820 (2000)

\title{
Quantum birth of the Universe in the varying speed of light cosmologies
}

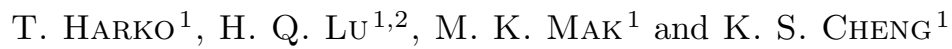

1 Department of Physics, The University of Hong Kong

Pokfulam, Hong Kong, P. R. China

2 Department of Physics, The Shanghai University - Shanghai, P. R. China

(received 20 August 1999; accepted in final form 6 January 2000)

PACS. 98.80.-k- Cosmology.

PACS. 98.80. Hw - Mathematical and relativistic aspects of cosmology; quantum cosmology.

\begin{abstract}
In the framework of the varying speed of light theory the Wheeler-DeWitt equation is considered in the minisuperspace approximation. The quantum potential is obtained and the tunneling probability is studied in both Vilenkin and Hartle-Hawking approaches.
\end{abstract}

Introduction. - Recently the cosmological implications of a variable speed of light have been considered in several papers. Varying speed of light (VSL) models proposed by Moffat [1, 2] and Albrecht and Magueijo [3], in which light is travelling faster in the early periods of the existence of the Universe, might solve the same problems as inflation. Hence they could become a valuable alternative explanation of the dynamics and evolution of our Universe and also provide a solution to the problem of the variation of the physical "constants" [4]. In its minimal formulation [3] the VSL theory starts from the variational principle

$$
S=\int \mathrm{d} x^{4} \sqrt{-g}\left[\frac{c^{4}\left(x^{i}\right)\left(R+2 \Lambda\left(x^{i}\right)\right)}{16 \pi G\left(x^{i}\right)}+L_{M}\right],
$$

which leads to the minimally modified Einstein field equations $[3,4]$

$$
R_{i}^{k}-\frac{1}{2} \delta_{i}^{k} R=\frac{8 \pi G\left(x^{j}\right)}{c^{4}\left(x^{j}\right)} T_{i}^{k}+\Lambda\left(x^{j}\right) \delta_{i}^{k} .
$$

Supposing that the gravitational constant $G$, the cosmological constant $\Lambda$ and the speed of light $c$ are independent fields, eq. (2) implies a matter creation process due to the nonconservativity of the energy-momentum tensor $T_{i}^{k}$. The particle number creation rate is determined by the (temporal) variations of $G, \Lambda$ and $c$ [5]. Hence in the framework of the minimal VSL model a self-consistent phenomenological description of the particle and entropy generation processes in the early Universe is obtained, describing the birth of a FRW-type Universe from a vacuum state [5]. On a classical level this scenario is very similar to models assuming that the Universe arose from nothing due to a quantum tunneling process [6] or as a result of some quantum fluctuations of the vacuum.

(c) EDP Sciences 
It is the purpose of the present letter to consider some quantum cosmological aspects of the minimal VSL theory with matter creation. In particular we shall investigate the problem of the quantum tunneling in theories with variable $G, \Lambda$ and $c$. The present letter is organized as follows. The classical VSL cosmological model is described in the following section. In the third section we obtain the corresponding Wheeler-DeWitt equation and the general properties of the quantum tunneling potential are considered. In the last section we conclude our results.

A classical VSL cosmological model. - In a preferred frame the VSL model FRW metric is given by $[1-3]$

$$
\mathrm{d} s^{2}=-c^{2}(t) \mathrm{d} t^{2}+a^{2}(t)\left[\frac{\mathrm{d} r^{2}}{1-k r^{2}}+r^{2}\left(\mathrm{~d} \theta^{2}+\sin ^{2} \theta \mathrm{d} \varphi^{2}\right)\right]
$$

with $k=0, \pm 1$. The corresponding gravitational field and particle production equations are [5]

$$
\begin{aligned}
& 3 \frac{\dot{a}^{2}}{a^{2}}+3 k \frac{c^{2}(t)}{a^{2}}=8 \pi G(t) \rho(t)+c^{2}(t) \Lambda(t), \\
& \dot{\rho}+3\left(\rho+\frac{p}{c^{2}(t)}\right) \frac{\dot{a}}{a}=-\frac{\rho(t)}{G(t)}\left[\frac{c^{2}(t) \dot{\Lambda}(t)}{8 \pi \rho(t)}+\dot{G}(t)-2 G(t) \frac{\dot{c}(t)}{c(t)}\right] .
\end{aligned}
$$

Equation (5), a consequence of the non-conservativity of the energy-momentum tensor, describes the generation of matter from vacuum. By assuming an equation of state of the form $p=(\gamma-1) \rho c^{2}(t), 1 \leq \gamma \leq 2$, and the phenomenological relations

$$
G=\frac{G_{0}}{a^{\alpha}}, \quad \Lambda=\frac{\Lambda_{0}}{a^{\beta}}, \quad c=\frac{c_{0}}{a^{\varepsilon}}, \quad \rho=\rho_{0} a^{n},
$$

with all $G_{0}, \Lambda_{0}, c_{0}, \rho_{0}, \alpha, \beta$ and $n$ non-negative, eq. (5) is identically satisfied if

$$
n=\alpha-\beta-2 \varepsilon \text { and } \beta=\frac{8 \pi \gamma G_{0} \rho_{0}}{A_{0} c_{0}^{2}},
$$

where we have denoted $A_{0}=\left(\Lambda_{0}+\frac{8 \pi G_{0} \rho_{0}}{c_{0}^{2}}\right) / 3$.

By using eqs. (4) and (7), we can express the general solution of the field equations in the following exact parametric form:

$$
\tau-\tau_{0}=\int \frac{A^{\varepsilon} \mathrm{d} A}{\sqrt{A^{2-\beta}-k}}, \quad \rho=\rho_{0} A_{0}^{\frac{n}{\beta-2}} A^{n}, \quad q=\varepsilon-\frac{2-\beta}{2\left(1-k A^{\beta-2}\right)} .
$$

In eq. (8) we have introduced the dimensionless scale factor $A$ and time $\tau$ by means of the transformations $a=A_{0}^{\frac{1}{\beta-2}} A$ and $\tau=c_{0} A_{0}^{\frac{\varepsilon+1}{2-\beta}} t$. The deceleration parameter is expressed as $q=-\frac{a \ddot{a}}{\dot{a}^{2}}$. For $k=0,-1$ the Universe started its evolution from a vacuum state with $a=0$ and $\rho=0$. For $k=+1$ the solution is physically defined only for values of $A^{2-\beta}>1$. The classical closed non-conservative Universe was born from a non-vacuum state with $a=a_{0}>0$ and $\rho=\rho_{0}>0$. Due to the variation of the speed of light and of the gravitational and cosmological constants, matter is generated from "nothing", and the energy density of the Universe is rapidly increasing. The variations of $\rho$ for a closed spherical geometry $(k=+1)$ and for various values of the parameters are represented in fig. 1 . The expansion of the closed FRW Universe is generally non-inflationary $(q>0)$ for all times but if the condition $\varepsilon+\frac{\beta}{2}<1$ is fulfilled, the Universe ends in an inflationary epoch. The density parameter $\Omega=\frac{3}{8 \pi G_{0} \rho_{0}} A_{0}^{\frac{\alpha-n}{\beta-2}}\left(1-k A^{\beta-2}\right)$ 


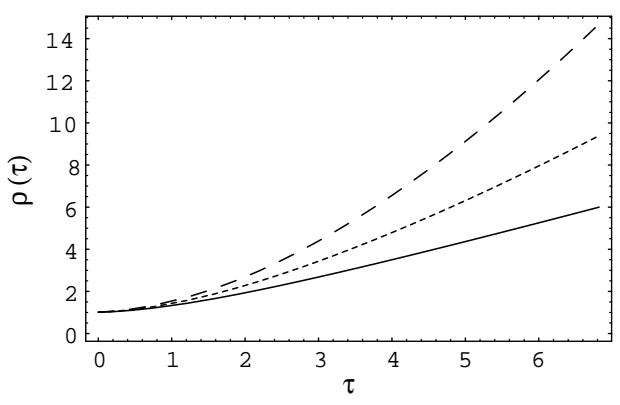

Fig. 1

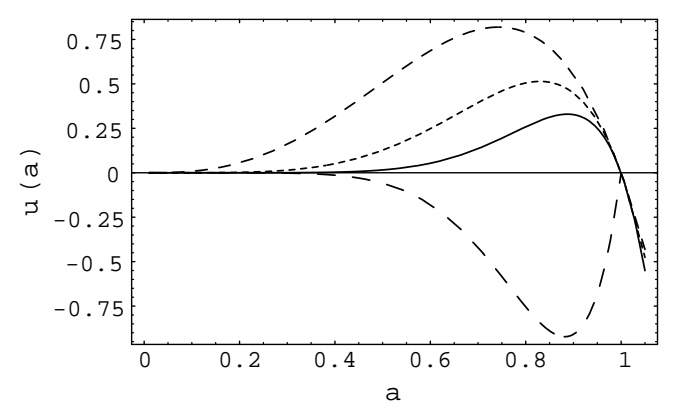

Fig. 2

Fig. 1 - Variation of the energy density $\rho$ (in the normalization $\rho_{0} A_{0}^{\frac{n}{\beta-2}}=1$ ) as a function of time $\tau$ for a closed FRW space-time $(k=+1)$ and for different values of the parameter $\alpha$ : $\alpha=3$ (full curve), $\alpha=3.25$ (dotted curve) and $\alpha=3.5$ (dashed curve) $\left(\beta=1, \varepsilon=\frac{1}{2}\right)$.

Fig. 2 - Variations of the quantum potential $u(a)=\frac{U}{U_{0}}$ as a function of the scale factor in the minisuperspace $(k=+1)$ for different values of the parameter $\alpha: \alpha=5$ (full curve), $\alpha=3.5$ (dotted curve), $\alpha=2.5$ (short-dashed curve) $\left(\beta=\frac{5}{4}, \varepsilon=\frac{1}{2}\right.$ ) and $\alpha=5, \beta=3$ and $\varepsilon=\frac{1}{2}$ (long-dashed curve).

is a free parameter that can be adjusted to match its value resulted from astronomical data. Recent observations show that $\Omega \sim 0.2-0.3$ [7], in contradiction with the prediction of the inflationary cosmology according to which the Universe will expand very close to the critical divide $\Omega=1$.

Quantum tunneling in the VSL cosmological models. - To formulate in the minisuperspace metric (3) the quantum version of the previous classical model, we shall write the total action $S=S_{G}+S_{M}$ in the form

$$
S=3 V_{3} \int \frac{c^{3}(a)}{8 \pi G(a)}\left((\varepsilon-1) a \dot{a}^{2}+k c^{2}(a) a-\frac{\Lambda(a) a^{3} c^{2}(a)}{3}-\frac{8 \pi G(a) \rho(a) a^{3}}{3}\right) \mathrm{d} t .
$$

To obtain (9) we have used the relation $\int \mathrm{d} x^{4} \sqrt{-g} \equiv \int V_{3} c(a) a^{3} \mathrm{~d} t$, eqs. (6) and we neglected a total derivative with respect to time. $V_{3}$ is the volume of the space-like hypersurfaces. Only the spherical three-space $(k=1)$ is of finite extent, with volume $V_{3}=2 \pi^{2}$ and, consequently, with finite action. The action (9) is similar to the actions proposed to describe $S O(3)$ invariant quasi-Riemannian models of gravity, with $\varepsilon$ a constant parametrizing the breaking of the local Lorentz symmetry [8]. In order to pass to the Hamiltonian formalism, conjugate momentum must be calculated. It is given by

$$
\Pi_{a}=\frac{\partial L}{\partial \dot{a}}=\frac{3 V_{3}(\varepsilon-1)}{4 \pi G(t)} c^{3}(a) a \dot{a} .
$$

From the last expression we obtain the canonical Hamiltonian $H=\Pi_{a} \dot{a}-L$,

$$
H=\frac{2 \pi G(a)}{3(\varepsilon-1) V_{3} c^{3}(a)} \frac{1}{a} \Pi_{a}^{2}-\frac{3 V_{3} c^{5}(a)}{8 \pi G(a)} k a+\frac{V_{3} c^{5}(a)}{8 \pi G(a)} \Lambda(a) a^{3}+V_{3} c^{3}(a) a^{3} \rho(a) .
$$

For $\varepsilon \ll 1$ the Hamiltonian constraint (11) $H=0$ gives eq. (4). The Dirac quantization of the Hamiltonian (11) consists in replacing the momentum according to the rule $\Pi_{a}^{2} \rightarrow-\hbar^{2} a^{-p} \frac{\partial}{\partial a}\left(a^{p} \frac{\partial}{\partial a}\right)$, where $p$ takes into account the factor ordering ambiguity. But this 
ambiguity is unimportant in the semiclassical approximation, so we shall take $p=0$. Taking into account that the action of the operator $\widehat{H}$ on the wave function of the Universe $\Psi$ gives zero, $\widehat{H} \Psi=0$, we obtain the Wheeler-DeWitt equation [9] describing the quantum dynamics of the Universe in the minimal VSL theory,

$$
\left(\frac{\mathrm{d}^{2}}{\mathrm{~d} a^{2}}-U(a)\right) \Psi(a)=0,
$$

where the quantum potential $U(a)$ is given by

$$
U(a)=\frac{9(1-\varepsilon) V_{3}^{2}}{16 \pi^{2} \hbar^{2}} \frac{c^{6}(a) a^{2}}{G^{2}(a)}\left[k c^{2}(a)-\frac{\Lambda(a) c^{2}(a) a^{2}}{3}-\frac{8 \pi G(a) a^{2}}{3} \rho(a)\right] .
$$

By using eqs. (6), (7) the quantum potential can be represented as

$$
U(a)=U_{0} a^{2(\alpha+1-4 \varepsilon)}\left(k-A_{0} a^{2-\beta}\right),
$$

where $U_{0}=\frac{9(1-\varepsilon) V_{3}^{2} c_{0}^{8}}{16 \pi^{2} \hbar^{2} G_{0}^{2}}$. This form of $U(a)$ presents some similarities with the potentials obtained in [10] for different physical (cosmological constant, decaying cosmological constant, scalar field and uniform density scalar field) models with the classical version of each model leading to inflation. For $\varepsilon=0$ and $\alpha=0$, we obtain exactly the same form as given by Norbury [10] for the quantum potential generated by a decaying cosmological constant. The variation of $U(a)$ is represented, for $k=+1$ and for different values of the constants $\alpha$, $\beta$ and $\varepsilon$ in fig. 2. For some classes of values of parameters the potential tends to zero for $a \rightarrow 0$, $U(0) \rightarrow 0$, then increases with increasing $a$, reaches a maximum and then turns over due to the second term in the bracket of eq. (14). By an appropriate choice of parameters the potential can also be displaced in the negative region. The maximum value of the quantum potential $U(a)$ is obtained for $a=a_{m}=\left(\frac{2(1+\alpha-4 \varepsilon)}{A_{0}(4+2 \alpha-\beta-8 \varepsilon)}\right)^{\frac{1}{2-\beta}}$. The zeros of $U(a)$ are given by $a=0$ and $a=a_{0}=A_{0}^{\frac{1}{\beta-2}}$. The shape and height of $U(a)$ are highly dependent on the numerical values of the constants describing the variations of the gravitational and cosmological constants and of the speed of light.

The evolution of the Universe can be viewed as the motion of a fictitious particle with zero energy and coordinate $a(t)$ in the potential given by eq. (14). The particle starts to the left of the potential and quantum mechanically there is a probability that the Universe can tunnel through the barrier and end up on the other side with a non-zero value of $a$. In the region $a<a_{0}$ the wave function becomes oscillatory while for $a>a_{0}$ the wave function exponentially decays.

In the classically allowed region the WKB solutions of eq. (12) are [11]

$$
\Psi_{ \pm}^{(1)}(a)=\exp \left[ \pm i \int_{a_{0}}^{a} \sqrt{-U\left(a^{\prime}\right)} \mathrm{d} a^{\prime} \mp i \frac{\pi}{4}\right]
$$

where $\Psi_{+}^{(1)}$ and $\Psi_{-}^{(1)}$ describe a contracting and expanding Universe. The component of the wave function describing the contracting Universe from the infinitely large side should be absent. This means that when the cosmological scale factor $a>a_{0}$ the Universe is under the expanding phase, $\Psi(a)=\Psi_{-}^{(1)}(a)$. Then, the WKB connection formula gives the under-barrier (classically forbidden or Euclidean region) solutions as

$$
\Psi^{(2)}(a)=\exp \left[\int_{a}^{a_{0}}\left|\sqrt{-U\left(a^{\prime}\right)}\right| \mathrm{d} a^{\prime}\right]
$$




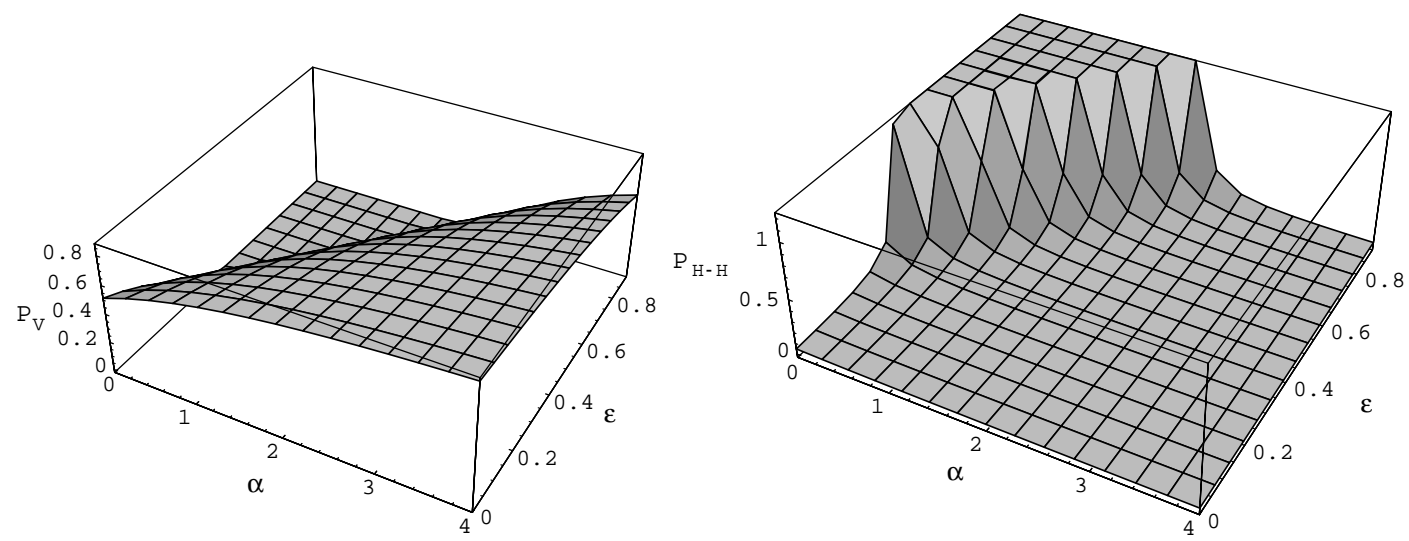

Fig. 3

Fig. 4

Fig. 3 - Variation of the tunneling probability $P_{V}=\left|\frac{\Psi\left(a_{0}\right)}{\Psi(0)}\right|^{2}$ as a function of the parameters $\alpha$ and $\varepsilon(\beta=0.01)$, with the wave function calculated by using the Vilenkin approach.

Fig. 4 - Variation of the tunneling probability $P_{\mathrm{H}-\mathrm{H}}$ as a function of the parameters $\alpha$ and $\varepsilon(\beta=0.01)$ in the Hartle-Hawking approach.

The nucleation probability (probability distribution for the initial values of $a$ in nucleating Universe) can be approximated as $[11,12]$

$$
P_{V}=\left|\frac{\Psi^{(2)}\left(a_{0}\right)}{\Psi^{(2)}(0)}\right|^{2} \sim \exp \left[-2 \int_{0}^{a_{0}}\left|\sqrt{-U\left(a^{\prime}\right)}\right| \mathrm{d} a^{\prime}\right] .
$$

In fig. 3 we have represented the variation of the tunneling probability (17) as a function of the parameters $\alpha$ and $\varepsilon(\beta=$ constant $=0.01)$. We have normalized the parameters by means of the relations $A_{0}=1$ and $U_{0}=1$. With this choice we also have $a_{0}=1$. The tunneling probability increases with increasing $\alpha$. For $\varepsilon=0$ and $\alpha=0$, this probability is, in our normalization, $P_{V}=0.51$, while for $\alpha=4$ and $\varepsilon=0.9$, the tunneling probability increases to $P_{V}=0.71$. But for fixed $\alpha$ and $\beta$ the tunneling probability decreases with increasing $\varepsilon$ (decreasing $c$ ).

The Hartle-Hawking (H-H) no-boundary wave function is given by the path integral [13]

$$
\Psi_{\mathrm{H}-\mathrm{H}}=\int[\mathrm{d} g] e^{-I_{\mathrm{eff}}(g)},
$$

where we integrate over all Euclidean-signature 4-metrics $g_{\mu \nu}$ defined on a 4-manifold $M_{4}$, that is, we take the 3 -manifold $M_{3}(h)$ with 3 -metrics $h_{i j}$ as a boundary. Hartle and Hawking [13] proposed as a cosmological initial condition that the manifold $M_{4}$ should have no other boundary than $M_{3}(h)$.

In order to determine $\Psi_{\mathrm{H}-\mathrm{H}}$, we first perform the Wick rotation $\tau=i t$ and consider a saddle-point approximation to the path integral (19), $\Psi_{\mathrm{H}-\mathrm{H}}(a) \propto e^{-\left.I_{\text {eff }}(a)\right|_{\text {saddle-point }}}$, where $I_{\text {eff }}$ is given by

$$
I_{\text {eff }}=4 \pi^{2} \int_{0}^{\tau} a^{\alpha+1-5 \varepsilon}\left(a^{2-\beta}-1\right) \mathrm{d} \tau
$$


and $a$ satisfies the classical equation of motion

$$
\frac{\mathrm{d} a}{\mathrm{~d} \tau}=a^{-\varepsilon}\left[\frac{1}{1-\varepsilon}\left(1-a^{2-\beta}\right)\right]^{\frac{1}{2}}, \quad a(0)=0 .
$$

In eqs. (19) and (20) we have used the same normalization of the parameters as for the Vilenkin wave function. In the limit $\alpha=\varepsilon=0$ and $\beta=$ constant, we recover the results of [13]. In fig. 4 we present the variation with $\alpha$ and $\varepsilon(\beta=$ constant $=0.01)$ of the tunneling probability $P_{\mathrm{H}-\mathrm{H}}$ obtained by using the Hartle-Hawking wave function. In this approach the tunneling probability is rapidly decreasing with increasing $\alpha$, but it increases with decreasing speed of light (increasing $\varepsilon$ ).

Conclusions. - In the present letter we have obtained the potential describing the quantum-mechanical evolution of the tunneling Universe in the framework of the VSL theory with matter creation. We also estimated the tunneling probability in both Vilenkin and Hartle-Hawking approaches. By allowing general variations of all the constants $c, G$ and $\Lambda$, a classical model describing matter creation is presented. The corresponding quantum potential in minisuperspace generalizes the tunneling inflationary potentials discussed in [10]. By appropriately choosing the arbitrary constants $\alpha, \beta$ and $\varepsilon$ describing the variations of the gravitational and cosmological constants and of the speed of light, respectively, we can reduce the height of the tunneling potential or even displace it in the negative region. If the potential is negative there is no possibility of tunneling anymore since a zero-energy system is always above the potential. The presence of a negative potential has a drastic effect on quantum creation scenarios as the forbidden region that is assumed to be tunneled through is no longer present. The classical singularity at zero scale factor is no longer isolated from the Universe by the quantum potential but instead classical evolution can start from arbitrarily small size.

This classical feature is also present in the original VSL phase transition scenario of Moffat $[1,2]$ and Albrecht and Magueijo [3], in which it is supposed there was a phase transition at time $t_{\mathrm{c}}$ when the speed of light changed from $c^{-}$to $c^{+}$. Sharp declines in $c$ always discharge any vacuum energy density into ordinary matter. In the cosmological solutions of this theory the condition of the positivity of $n$ from eq. (7) is not imposed, hence the energy density of the matter is generally decreasing. In the phase transition model the form of the quantum potential can also be obtained in the form (13). If the condition $c^{2}(a)<\frac{8 \pi G(a) a^{2} \rho(a) / 3}{k-\Lambda(a) a^{2} / 3}$ holds, the potential is displaced in the negative region. Therefore if the speed of light changed the dynamics of the very early Universe was classical. This result, obtained in the present paper from a quantum approach, is consistent with the result of Barrow and Magueijo [14] obtained in the framework of a classical phase transition VSL model. By using for the dynamics of $c$ a model inspired from dilaton theories, they have found that, as we go back in time, $\frac{t}{t_{P}}$ (where $t_{P}=\left(G h / c^{5}\right)^{1 / 2}$ is the Planck time) increases and hence the Universe does not have a quantum origin. As a result of a rapid change of the speed of light the very early Universe became very classical, due to the annihilation of the positive component of the quantum potential. Because the forbidden region is absent, the wave function describing the quantum evolution of the Universe will be purely oscillatory.

The classical Universe born as a result of the VSL process is in a non-inflationary stage, with still varying $c, \Lambda$ and $G$. The rapid change of the speed of light during the early evolution of the Universe can also solve the flatness and horizon problem [1-4]. The speed of light is the only fundamental physical constant having this property, a change in the gravitational constant $G$ does not solve the cosmological problems solved by inflation [3]. Future work will be devoted to find exact solutions of the Wheeler-DeWitt equation (15) and to extend the 
present analysis to open and flat geometries and to non-minimal VSL theories.

$$
* * *
$$

The authors are very grateful to the referees for many helpful comments, which helped to improve the manuscript. This paper is supported by a RGC grant of Hong Kong Government.

\section{REFERENCES}

[1] Moffat J. W., Int. J. Mod. Phys. D, 2 (1993) 351.

[2] Moffat J. W., preprint astro-ph/9811390 (1998).

[3] Albrecht A. and Magueijo J., Phys. Rev. D, 59 (1999) 043516.

[4] Barrow J. D., Phys. Rev. D, 59 (1999) 043515; Barrow J. D. and Magueijo J., Phys. Lett. B, 443 (1999) 104; Bekenstein J. D., Phys. Rev. D, 25 (1982) 1527; Barrow J. D. and Magueijo J., Phys. Lett. B, 447 (1999) 246; Class. Quantum Grav., 16 (1999) 1435; Clayton M. A. and Moffat J. W., Phys. Lett. B, 460 (1999) 263; Coule D. H., Mod. Phys. Lett. A, 14 (1999) 2437; Barrow J. D. and O'Toole C., preprint astro-ph/9904116 (1999); Avelino P. P. and Martins C. J. A. P., Phys. Lett. B, 459 (1999) 468.

[5] Harko T. and Mak M. K., Class. Quantum. Grav., 16 (1999) 2741.

[6] Atkatz D. and Pagels H., Phys. Rev. D, 25 (1982) 2065; Vilenkin A., Phys. Rev. D, 27 (1983) 2848; Vilenkin A., Phys. Rev. D, 39 (1989) 1116; Coule D. H. and Martin J., preprint gr-qc/ 9905056 (1999).

77] Ratra B. and Peebles P. J. E., Phys. Rev. D, 37 (1988) 3406; Bahcall N. A., Lubin L. M. and Dorman V., Astrophys. J., 447 (1995) L81; 169.

[8] Weinberg S., Phys. Lett. B, 138 (1984) 47; Gasperini M., Phys. Lett. B, 163 (1985) 84.

[9] DeWitt B. S., Phys. Rev., 160 (1967) 1113.

[10] Norbury J. W., Phys. Lett. B, 433 (1998) 263.

[11] Vilenkin A., Phys. Rev. D, 50 (1994) 2581.

[12] Linde A. D., Lett. Nuovo Cimento, 39 (1984) 401.

[13] Hartle J. B. and Hawking S. W., Phys. Rev. D, 28 (1983) 2960; Hawking S. W., Nucl. Phys. B, 239 (1984) 257.

[14] Barrow J. D. and Magueijo J., preprint astro-ph/9907354 (1999). 